\title{
Synthesis, identification and thermal study of some new inorganic polymers based on bis-dithiocarbamate ligands with silicone, tellurium and some transition metals
}

\author{
Síntesis, identificación y estudio térmico de algunos \\ nuevos polímeros inorgánicos basados en ligandos de bis- \\ ditiocarbamato con silicona, teluro $y$ algunos metales de \\ transición
}

Author:

Nasir $\mathbf{H ~ M}^{1}$

Saki T A ${ }^{2}$

Al-Luaibi M Y $\mathbf{Y}^{3}$

\section{SCIENTIFIC RESEARCH}

How to cite this paper:

Nasir, H. M., Saki, T. A., Al-Luaibi, M. Y., Synthesis, identification and thermal study of some new inorganic polymers based on bis-dithiocarbamate ligands with silicone, tellurium and some transition metals. Innovaciencia. 2019; 7 (1): 1-13.

http://dx.doi.org/10.15649/2346075X.507

Reception date:

Received: 28 January 2019

Accepted: 19 May 2019

Published: 25 October 2019

Keywords:

bis-Dithiocarbamate ligands; Epoxy resins; Si; Te; Inorganic polymer

\section{ABSTRACT}

Introduction: In recent years, there has been considerable interest in dithiocarbamate complexes because of its diverse biological roles, very few reports have been made on polymeric bis- dithiocarbamate compounds with carbon chain of $\mathrm{n}$-propyl or hexamethelene with transition metals in addition of the absence of any report of organosilicone or tellurium halides with such compounds Our interest in this report based on the preparation of new series of polymers with an expected activity as a fungi side compounds followed by the using of prepared amino compound as a hardners for epoxy paints Materials and Methods: A new polymers of the general structures - $\left(\mathrm{MS}_{2} \mathrm{CNH}\left(\mathrm{CH}_{2}\right)_{6} \mathrm{NHCS}_{2} \mathrm{M}-\right)_{\mathrm{n}}$ where $[\mathrm{M}=\mathrm{Cu}, \mathrm{Cd}$, $\mathrm{Mn}$ and $\mathrm{Zn}]$ and $-\left(\mathrm{M}(\mathrm{R}){ }_{2} \mathrm{~S}_{2} \mathrm{CNH}\left(\mathrm{CH}_{2}\right)_{\mathrm{n}}^{2} \mathrm{NHCS}_{2} \mathrm{MR}_{2}-\right)_{\mathrm{m}}^{\mathrm{n}}$ where $[\mathrm{M}=\mathrm{Si}$, $\mathrm{R}=\mathrm{CH}_{3}, \mathrm{n}=2,3$ and $6 ; \mathrm{M}=\mathrm{Te}, \mathrm{R}=\mathrm{Br}, \mathrm{n}=2,3$ and $6 ; \mathrm{M}=\mathrm{Co}, \mathrm{Ni} \mathrm{R}=\mathrm{Cl}$; $\mathrm{n}=6]$ have been prepared by reaction of $\mathrm{MX}_{2}$ where $\mathrm{M}=\mathrm{Ni}, \mathrm{Co}, \mathrm{Cd}, \mathrm{Mn}$ or $\mathrm{Zn}, \mathrm{X}=\mathrm{Cl} ; \mathrm{M}=\mathrm{Cu}, \mathrm{X}=\mathrm{SO}_{4}$, and dimethyl dichloro silane, tellurium tetrabromide with the corresponding sodium salts of bis-dithiocarbamate ligands. Results and Discussion: Dimethyldichloro silane is a very sensitive material to $\mathrm{O}-\mathrm{H}$ group, in addition to that, $\mathrm{TeBr}_{4}$ decomposed rapidly in water so, a series solvents may be useful with such sensitive chemicals to water, in the other hand, dithiocarbamate ligands which is usually prepared in aqueous and alcoholic solution, must be prepared and isolated carefully to apply the other steps of synthesis using a chloroform solution as a solvent. It seems for the first view for dithiocarbamato ligands it may act as a bi dentate ligands using two sulphur donating atoms that is clearly appeared in common complexes such as diethyl dithiocarbamato or pipyridyl, morpholino dithiocarbamato with representation metal elements, even in such type of elements a sulfur bridges may formed. In this study, it showed clearly that Zinc and Cadimium polymers are diamagnetic polymers that is mean that these polymers are with oxidation state equal to (II) and a tetrahedral configuration Conclusions: The study showed that the new silicone polymers act as a stable polymers compared with others. All new polymers are of a high stability with large values of char contain compared with commercial epoxy. Among silicone polymers, the polymer with carbon chain equal to 3 is more stable than that with 2 carbon atoms while the silicone polymer with 6 carbon atoms is the less stable one, may thermal treatment caused decomposition combined..

\footnotetext{
Department of Chemistry, College of Science, University of Basrah, Basrah, Iraq

Department of Chemistry, College of Science, University of Basrah, Basrah, Iraq

Department of Chemistry, College of Science, University of Basrah, Basrah, Iraq, majeedalluaibi7@gmail.com.
} 


\section{INTRODUCTION}

In recent years, there has been considerable interest in dithiocarbamate complexes because of its diverse biological roles, very few reports have been made on polymeric bis- dithiocarbamate compounds with carbon chain of n-propyl or hexamethelene with transition metals in addition of the absence of any report of organosilicone or tellurium halides with

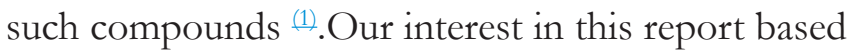
on the preparation of new series of polymers with an expected activity as a fungi side compounds followed by the using of prepared amino compound as a hardners for epoxy paints ${ }^{(2)}$. The aim of this work is to prepare a new series of polymers based on dithiocarbamate of silicon and tellurium and some first-row transition metals in hope of finding a new coating epoxide with an anti-fungal property so may use in agriculture studies.

\section{MATERIALS AND METHODS EXPERI-} MENTAL

\section{a) Physical measurements}

The FTIR spectra were recorded in the range 4000$400 \mathrm{~cm}^{-1}$ on a FTIR-8400 SHIMADZO using $\mathrm{KBr}$ discs. ${ }^{1} \mathrm{H}$ NMR spectra were measured on a Bruker at $400 \mathrm{MHz}$ in the department of chemistry / University of the Westem Cape, South Africa.

Thermal analysis of the polymers was measured at Perkin Elmer Pyris TGA in the department of chemistry /University of the Westem Cape, South Africa, while Epoxy Samples were recorded on TGA-Q50(Made in USA) in the department of chemistry / college of science /Basrah University / IRAQ. DSC measurements were recorded on DSC60, DIFFRENTIAL SCANNING CALOMETER /SHIMADZO, at the centre of polymeric researches /BASRAH/IRAQ. Atomic Absorptions were recorded on AA-7000- ATOMIC ABSORPTION SPECTROMETER /SHIMADZO, in the Marine Science Laps/IRAQ. C.H.N. analysis was recorded on a Varior EL Cube Elemental analysis C.H.N.in the department of chemistry / University of the Westem Cape, South Africa. Melting points were measured by a Gallenkamp melting point apparatus and are uncorrected.

\section{b- Chemicals :}

Cadimium Chloride $\left(\mathrm{CdCl}_{2}\right)$, Zinc Chloride $\left(\mathrm{ZnCl}_{2}\right)$, Manganese Chloride $\left(\mathrm{MnCl}_{2} \cdot 4 \mathrm{H}_{2} \mathrm{O}\right)$, Cobaltous Chloride $\left(\mathrm{CoCl}_{2} .6 \mathrm{H}_{2} \mathrm{O}\right)$, Nickel Chloride $\left(\mathrm{NiCl}_{2}\right.$ $\left..6 \mathrm{H}_{2} \mathrm{O}\right)$,Cupper -Sulfate $\left(\mathrm{CuSO}_{4} .5 \mathrm{H}_{2} \mathrm{O}\right)$, dimethyl dichloro silane $\left(\mathrm{CH}_{3}\right)_{2} \mathrm{SiCl}_{2}$, Tellurium powder, Bromine, organo diamines, carbon disulfphide $\left(\mathrm{CS}_{2}\right)$ and common solvents were provided from $\mathrm{RDH}$ company and used without other purification.

$\mathrm{TeBr}_{4}$ were prepared by slowly addition of a solution of 2 moles of bromine in chloroform to 1 mole of tellurium powder on a period of about $1 \mathrm{~h}$. until all gray tellurium powder disappeared, further stirring to about $3 \mathrm{hrs}$, filtered and washing several times with chloroform.

\section{c- Synthesis:}

Sodium (ethylene bis-dithiocarbamate) bydrate (I), Sodium (propylene bis-dithi-ocarbamate) bydrate (II) and sodium (hexamethylene bis-dithiocarbamate) bydrate (III) ligands were prepared by the reaction ethanolic solution of the corresponding amine with carbon disulphide (mole ratio1:2) in aqueous solution of sodium hydroxide according to a general literature method ${ }^{(3)}$. The products were recrystallized from methanol verified by their melting points, FTIR and elemental analysis and other techniques.

\section{Poly[ethylene bis-dithiocarbamato bis(Telluri- um(IV) dibromide)] hydrate (9)}

Tellurium tetrabromide (8.94 g; 0.02 mole ) in 25 $\mathrm{ml}$ of chloroform was added to a stirred solution of sodium (ethylene bis-dithiocarbamato) ( $2.56 \mathrm{~g}$; 0.01 mole) in $25 \mathrm{ml}$ of chloroform the mixture was stirred for $3 \mathrm{~h}$. after which the solvent was removed under reduced pressure, a green precipitate formed , the product washed several times with chloroform followed by addition of 3 portions of deionized 
water, filtered to sodium bromide and dried .

Poly[ethylene bis-dithiocarbamato tetrakis-dimethyl Silane)] hydrate (10)

Dimethyl dichloro silane $(2.4 \mathrm{ml} ; 0.02$ mole $)$ in 10 $\mathrm{ml}$ of chloroform was added to a stirred solution of sodium (ethylene Bis-dithiocarbamato)(2.56 g ; 0.01 mole) in $25 \mathrm{ml}$ of chloroform, the mixture was stirred to $3 \mathrm{~h}$. after which the solvent was removed under reduced pressure, solid precipitate formed, the product washed several times with chloroform followed by addition of 3 portions of de ionized water, filtered to remove sodium chloride, the product was dried to obtain a yellow precipitate .

Poly [propelene bis-dithiocarbamato bis-(Tellurium(IV) dibromide)] hydrate (11)

The new polymer has been prepared as previously described in the synthesis of polymer (1) by the addition of Tellurium tetrabromide $(8.94 \mathrm{~g} ; 0.02$ mole ) in $25 \mathrm{ml}$ to a stirred solution of sodium (propelene bis-dithiocarbamato)( $2.70 \mathrm{~g} ; 0.01 \mathrm{~mole})$ in $25 \mathrm{ml}$ of chloroform. A light green precipitate was obtaining.

Poly [propelene bis-dithiocarbamato tetrakis-dimethyl Silane)] hydrate (12)

The new polymer has been prepared as previously described in the synthesis of polymer (2) by the addition of dimethyl dichloro silane $(1.2 \mathrm{ml} ; 0.01$ mole ) in $10 \mathrm{ml}$ of chloroform was added to a stirred solution of sodium (propelene bis-dithiocarbamato) ( $2.70 \mathrm{~g} ; 0.01 \mathrm{~mole}$ ) in $25 \mathrm{ml}$ of chloroform . A deep red precipitate was obtaining.

\section{Poly [hexamethylenebis-dithiocarbamato diTel- lurium (IV) dibromide] hydrate(1)}

The new polymer has been prepared as previously described in the synthesis of polymer (1) by the addition of Tellurium tetrabromide (8.94 g; 0.02 mole) in $25 \mathrm{ml}$ to a stirred solution of sodium (propelene bis-dithiocarbamato) ( $2.70 \mathrm{~g}$; $0.01 \mathrm{~mole})$ in $25 \mathrm{ml}$ of chloroform. An orange precipitate was obtaining.

Poly [hexamethylenete bis-dithiocarbamato tetrakis-dimethyl Silane] hydrate (2)

The new polymer has been prepared as previously described in the synthesis of polymer (2) by the addition of dimethyl dichloro silane $(2.4 \mathrm{ml} ; 0.02$ mole ) in $10 \mathrm{ml}$ of chloroform was added to a stirred solution of sodium (propelene bis-dithiocarbamato) (3.12 $\mathrm{g} ; 0.01 \mathrm{~mole}$ ) in $25 \mathrm{ml}$ of chloroform . An orange precipitate was obtaining.

Poly [hexamethylenebis-dithiocarbamato diZinc(II)] hydrate (3), Poly[hexamethylene bis-dithiocarbamato dichlorodiNickel(II) ] hydrate(4), Poly[hexamethylene bis-dithiocarbamato dichlorodiCobalt(II) ] hydrate(5), Poly[hexamethylene bis-dithiocarbamato diCadimium(II) ] hydrate (6),Poly -[hexamethylene bis-dithiocar -bamato dichlorodiManganese(II) ]hydrate (7), Poly[hexamethylene bisdithiocarba-mato diCupper(II) ] hydrate(8). The new polymers above have been prepared by the addition of aqueous solutions of transition metal salts $(0.02$ mole $)$ to a stirred solution of sodium (propelene bis-dithiocarbamato) ( $3.12 \mathrm{~g} ; 0.01$ mole) in $25 \mathrm{ml}$ of ethanol, the mixture was stirred for $3 \mathrm{~h}$. after which the solvent was removed under reduced pressure, a solid precipitate formed, the product washed several times with ethanol followed by addition of 3 portions of de ionized water , filtered to remove sodium bromide then dried. All preparations are shown in Scheme 1 below. 


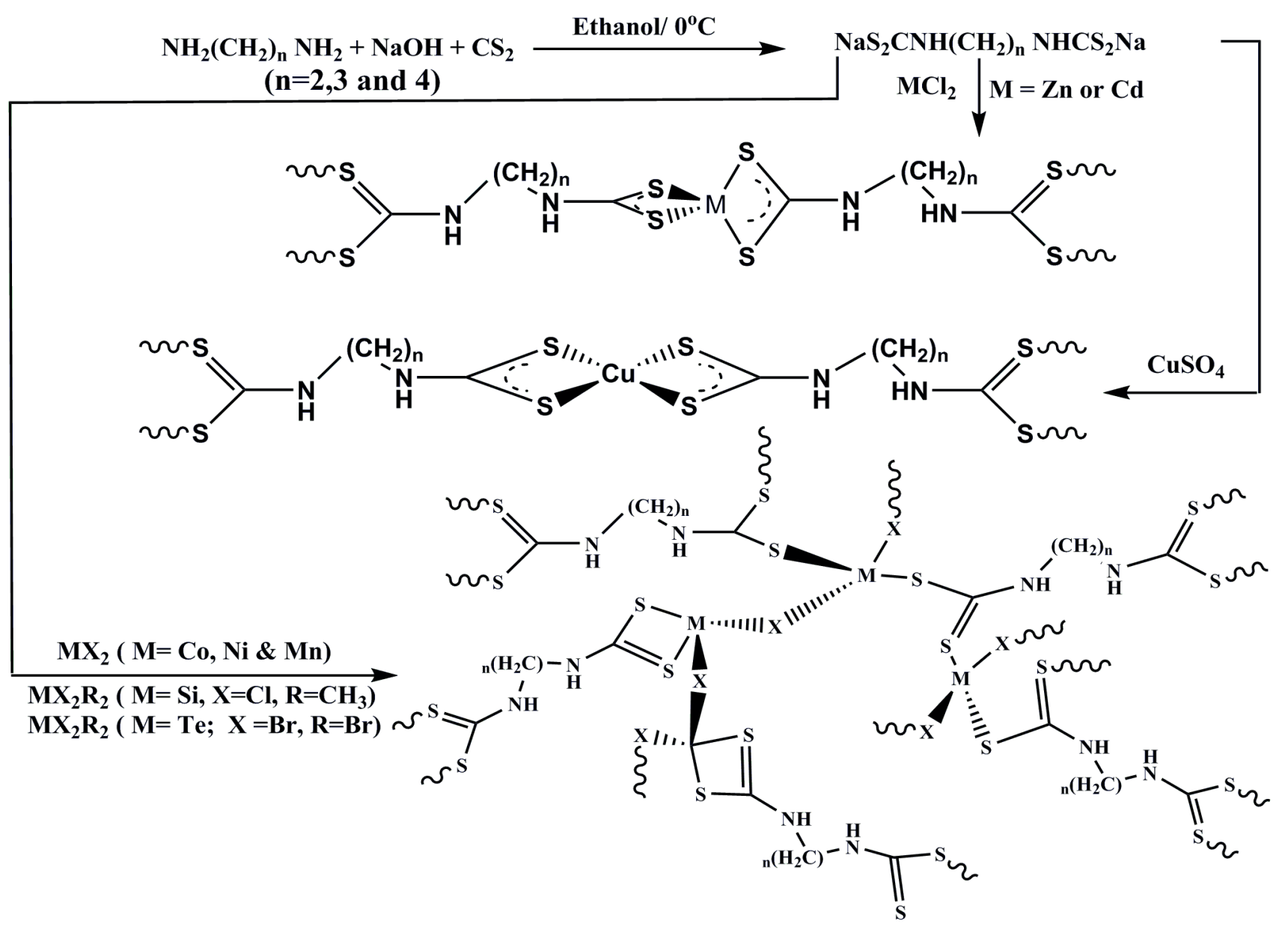

Scheme 1. The preparation of new ligands and polymers

\section{RESULTS AND DISCUSSION}

The main purpose for this study is to prepare new tellurium, selenium and some first-row transition metals polymers. The new polymers may have some biological activity as it is well known that ditiocarbamate derivatives have some important biological activity ${ }^{(4)}$.Dimethyldichloro silane is a very sensitive material to $\mathrm{O}-\mathrm{H}$ group, in addition to that, $\mathrm{TeBr}_{4}$ decomposed rapidly in water so, a series solvents may be useful with such sensitive chemicals to water, in the other hand, dithiocarbamate ligands which is usually prepared in aqueous and alcoholic solution, must be prepared and isolated carefully to apply the other steps of synthesis using a chloro form solution as a solvent. It seems for the first view for dithiocarbamato ligands it may act as a bi dentate ligands using two sulfur donating atoms that is clearly appeared in common complexes such as diethyl dithiocarbamato or pipyridyl, morpholino dithiocarbamato with representation metal elements, even in such type of elements a sulfur bridges may formed $(5)$.

It is worth noting that the configuration of transition metal in these polymers are either square planner or tetrahedral due to presence of d-orbitals. All prepared polymers are insoluble in common organic solvents. They are soluble in DMSO. A proposed structure based on elemental analysis and magnetic susceptibility shown in Table 1 and $2 \mathrm{~b}$. 
Table 1. Physical properties of new polymers based on bis-dithiocarbamate

\begin{tabular}{|c|c|c|c|c|c|c|c|c|}
\hline \multirow[b]{2}{*}{$\begin{array}{l}\text { Compd. } \\
\text { no. }\end{array}$} & \multirow[b]{2}{*}{ Structure } & \multicolumn{3}{|c|}{ Physical properties } & \multicolumn{4}{|c|}{ C.H.N } \\
\hline & & $\begin{array}{l}\mathrm{M} \cdot \mathrm{p} \\
{ }^{\circ} \mathrm{C}\end{array}$ & Color & $\begin{array}{l}\text { Yeild } \\
\%\end{array}$ & $\mathrm{C} \%$ & $\mathrm{H} \%$ & $\begin{array}{l}\mathrm{N} \\
\%\end{array}$ & $\mathrm{M} \%$ \\
\hline III & $\mathrm{NaS}_{2} \mathrm{CNH}\left(\mathrm{CH}_{2}\right)_{6} \mathrm{NHCS}_{2} \mathrm{Na} .4 \mathrm{H}_{2} \mathrm{O}$ & 80 & white & 86 & $\begin{array}{c}24.99 \\
24.2\end{array}$ & $\begin{array}{r}5.77 \\
4.7\end{array}$ & $\begin{array}{c}7.29 \\
6.7\end{array}$ & \\
\hline 1 & $\mathrm{TeBr}_{2} \mathrm{~S}_{2} \mathrm{C}\left(\mathrm{CH}_{2}\right)_{2} \mathrm{NHCS}_{2} \mathrm{TeBr}_{2} \cdot 4 \mathrm{H}_{2} \mathrm{O}$ & $100(d)$ & orange & 75 & & & & \\
\hline 2 & $\mathrm{TeBr}_{2} \mathrm{~S}_{2} \mathrm{C}\left(\mathrm{CH}_{2}\right)_{3} \mathrm{NHCS}_{2} \mathrm{TeBr}_{2} \cdot 4 \mathrm{H}_{2} \mathrm{O}$ & $82(d)$ & orange & 65 & & & & \\
\hline 3 & $\mathrm{TeBr}_{2} \mathrm{~S}_{2} \mathrm{C}\left(\mathrm{CH}_{2}\right)_{6} \mathrm{NHCS}_{2} \mathrm{TeBr}_{2} .4 \mathrm{H}_{2} \mathrm{O}$ & $70(d)$ & brown & 91 & $\begin{array}{c}10.52 \\
9.5\end{array}$ & $\begin{array}{l}2.43 \\
2.90\end{array}$ & $\begin{array}{l}3.07 \\
2.90\end{array}$ & \\
\hline 4 & $\begin{array}{c}\mathrm{Si}\left(\mathrm{CH}_{3}\right)_{2} \mathrm{~S}_{2} \mathrm{CNH}\left(\mathrm{CH}_{2}\right)_{2} \mathrm{NHCS}_{2} \mathrm{Si}\left(\mathrm{CH}_{3}\right)_{2} \\
\left(\mathrm{Si}\left(\mathrm{CH}_{3}\right)_{2}\right)_{2} \mathrm{H}_{2} \mathrm{O}\end{array}$ & $260(d)$ & yellow & 72 & & & & \\
\hline 5 & $\begin{array}{c}\mathrm{Si}\left(\mathrm{CH}_{3}\right)_{2} \mathrm{~S}_{2} \mathrm{CNH}\left(\mathrm{CH}_{2}\right)_{3} \mathrm{NHCS}_{2} \mathrm{Si}\left(\mathrm{CH}_{3}\right)_{2} \\
\left(\mathrm{Si}\left(\mathrm{CH}_{3}\right)_{2}\right) \cdot 2 \mathrm{H}_{2} \mathrm{O}\end{array}$ & 80 & $\begin{array}{l}\text { Light } \\
\text { gray }\end{array}$ & 74 & $\begin{array}{l}31.67 \\
31.50\end{array}$ & $\begin{array}{l}7.36 \\
6.13\end{array}$ & $\begin{array}{l}5.68 \\
4.97\end{array}$ & \\
\hline 6 & $\begin{array}{c}\mathrm{Si}\left(\mathrm{CH}_{3}\right)_{2} \mathrm{~S}_{2} \mathrm{CNH}\left(\mathrm{CH}_{2}\right)_{6} \mathrm{NHCS}_{2} \mathrm{Si}\left(\mathrm{CH}_{3}\right)_{2}\left(\mathrm{Si}\left(\mathrm{CH}_{3}\right)_{2}\right)_{2} \\
.2 \mathrm{H}_{2} \mathrm{O}\end{array}$ & $180(d)$ & gray & 96 & $\begin{array}{c}35.91 \\
42.5\end{array}$ & $\begin{array}{l}7.91 \\
7.2\end{array}$ & $\begin{array}{l}5.23 \\
13.4\end{array}$ & \\
\hline 7 & $\mathrm{ZnS}_{2} \mathrm{CNH}\left(\mathrm{CH}_{2}\right)_{6} \mathrm{NHCS}_{2} \mathrm{Zn} .4 \mathrm{H} 2 \mathrm{O}$ & $220(d)$ & white & 94 & $\begin{array}{c}23.79 \\
23.8\end{array}$ & $\begin{array}{c}5.49 \\
4.3\end{array}$ & $\begin{array}{c}6.94 \\
7.0\end{array}$ & $\begin{array}{c}27.86 \\
27.9\end{array}$ \\
\hline 8 & $\mathrm{CoCl}_{2} \mathrm{~S} 2 \mathrm{CNH}(\mathrm{CH} 2)_{6} \mathrm{NHCS}_{2} \mathrm{CoCl}_{2} \cdot 4 \mathrm{H}_{2} \mathrm{O}$ & $180(d)$ & $\begin{array}{l}\text { Dark } \\
\text { green }\end{array}$ & 89 & & & & $\begin{array}{c}20.11 \\
19.8\end{array}$ \\
\hline 9 & $\mathrm{NiCl}_{2} \mathrm{~S}_{2} \mathrm{CNH}\left(\mathrm{CH}_{2}\right)_{6} \mathrm{NHCS}_{2} \mathrm{NiCl}_{2} .4 \mathrm{H}_{2} \mathrm{O}$ & $100(d)$ & green & 98 & $\begin{array}{c}16.08 \\
17.5\end{array}$ & $\begin{array}{c}3.71 \\
4.6\end{array}$ & $\begin{array}{c}4.69 \\
4.9\end{array}$ & $\begin{array}{c}19.64 \\
19.8\end{array}$ \\
\hline 10 & $\mathrm{CdS}_{2} \mathrm{CNH}\left(\mathrm{CH}_{2}\right)_{6} \mathrm{NHCS}_{2} \mathrm{Cd} .4 \mathrm{H}_{2} \mathrm{O}$ & $295(d)$ & yellow & 90 & & & & $\begin{array}{c}39.91 \\
39.7\end{array}$ \\
\hline 11 & $\mathrm{CuS}_{2} \mathrm{CNH}\left(\mathrm{CH}_{2}\right)_{6} \mathrm{NHCS}_{2} \mathrm{Cu} .4 \mathrm{H}_{2} \mathrm{O}$ & $60(\mathrm{~d})$ & $\begin{array}{l}\text { Light } \\
\text { green }\end{array}$ & 90 & & & & $\begin{array}{c}27.30 \\
27.7\end{array}$ \\
\hline 12 & $\mathrm{MnCl}_{2} \mathrm{~S}_{2} \mathrm{CNH}\left(\mathrm{CH}_{2}\right)_{6} \mathrm{NHCS}_{2} \mathrm{MnCl}_{2} \cdot 4 \mathrm{H}_{2} \mathrm{O}$ & $110(\mathrm{~d})$ & $\begin{array}{c}\text { Deep } \\
\text { red }\end{array}$ & 89 & & & & $\begin{array}{c}24.50 \\
24.6\end{array}$ \\
\hline
\end{tabular}

\section{MAGNETIC SUSCEPTIBILITY}

In this study it showed clearly that Zinc and Cadimium polymers are a diamagnetic polymer that is mean that these polymers are with oxidation state equal to (II) and a tetrahedral configuration. Cobalt(II) polymer gave a magnetic susceptibility with $(\mu=3.85$ B.M.) with oxidation state equal to 2 and $\left(d^{7}\right)$ system with three unpaired electrons that is mean that the configuration of this polymer is a tetrahedral with electron configuration of $\left(\mathbf{e}^{4} \mathbf{t}_{2}{ }^{3}\right)$ while Nickel complex showed ( $\mu=2.37$ B.M.) with oxidation state equal to 2 and $\left(\mathrm{d}^{8}\right)$ system with two unpaired electrons that is mean that the configuration of this polymer is also tetrahedral with electron configuration of $\left(\mathbf{e}^{4} \mathbf{t}_{2}{ }^{4}\right)$. See Scheme 1 and Table $2 \mathrm{~b}$.

\section{INFRARED SPECTRA}

The characteristic stretching peaks in the FTIR spectra of the dithiocarbamate have been assigned. The $\mathrm{NH}$ unsymmetrical vibrations bands which were observed between 3167 and $3350 \mathrm{~cm}^{-1}$ as a strong 
Table 2a. Infrared spectra for new compounds

\begin{tabular}{cccccc}
\hline \multirow{2}{*}{ Compd. No. } & \multicolumn{3}{c}{ Infrared spectra $\left(\mathrm{cm}^{-1}\right)$} & C-S & M-S \\
\hline III & 3167 & 2929,2858 & 1512 & 947 & 530 \\
1 & 3167 & 2931,2860 & 1514 & 943 & 624 \\
2 & 3190 & 2929,2856 & 1516 & 941 & 667 \\
3 & 3236 & 2927,2856 & 1512 & 952 & 619 \\
4 & 3213 & 2929,2872 & 1525 & 947 & 667 \\
5 & 3232 & 2931,2856 & 1514 & 954 & 615 \\
6 & 3290 & 2927,2858 & 1514 & 956 & 626 \\
7 & 3221 & 2935,2858 & 1506 & 1080 & 729 \\
8 & 3350 & 2935,2858 & 1506 & 1109 & 624 \\
9 & 3290 & 2927,2858 & 1514 & 956 & 626 \\
10 & 3221 & 2935,2858 & 1506 & 1080 & 729 \\
11 & 3350 & 2935,2858 & 1506 & 1109 & 624 \\
12 & 3167 & 2935,2858 & 1506 & 1109 & 624 \\
\hline
\end{tabular}

band confirm with proposed structures. The wide variation in position gave another evidence of coordination with different metals. The sodium salts of dithiocarbamato show a medium peak at $530 \mathrm{~cm}^{-1}$
, while M-S vibration frequencies appeared at 616$730 \mathrm{~cm}^{-1}$. A very strong bands appeared in the range 930- $1108 \mathrm{~cm}^{-1}$ which is a characteristic band for C-S stretching vibrations. $\mathrm{C}-\mathrm{H}_{\text {alef }}$ and $\mathrm{C}-\mathrm{N}$ also appeared

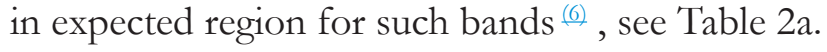

Table 2b. Uv-visible spectra and Magnetic susceptibility for new compounds

\begin{tabular}{ccccc}
\hline \multicolumn{5}{c}{ UV-Visible spectra $\mathrm{nm}\left(\mathrm{cm}^{-1}\right)$} \\
Compd. No. & III & II & I & $\mu_{\text {eff }}$ (B.M) \\
\hline III & $369(27,100)$ & $293(34,129)$ & $228(43,859)$ & - \\
1 & $370(27,027)$ & $277(36,101)$ & $228(43,859)$ & - \\
2 & $369(27,100)$ & $293(34,129)$ & $228(43,859)$ & - \\
3 & $377(36,101)$ & $358(27,933)$ & $296(33.557)$ & dia. \\
4 & $396(252,525)$ & $283(35,336)$ & $232(43,103)$ & 2.37 \\
5 & $377(26,525)$ & $273(36,630)$ & $230(43,478)$ & 3.85 \\
6 & $373(26,809)$ & $281(35,587)$ & $231(43,290)$ & dia. \\
7 & $376(26,595)$ & $273(36,630)$ & $230(43,478)$ & 1.694 \\
8 & $373(26,809)$ & $283(35,336)$ & $231(43,290)$ & 0.884 \\
9 & $373(26,809)$ & $281(35,587)$ & $231(43,290)$ & - \\
10 & $376(26,595)$ & $273(36,630)$ & $230(43,478)$ & - \\
11 & $373(26,809)$ & $283(35,336)$ & $231(43,290)$ & - \\
12 & $373(26,809)$ & $283(35,336)$ & $231(43,290)$ & - \\
\hline
\end{tabular}




\section{${ }^{1}$ H NMR SPECTRA:}

${ }^{1} \mathrm{H}$ NMR data also confirm structures (1-12). The $\mathrm{CH}_{2}\left(1 \& 1^{\prime}\right)$ proton as a quartet bands are observed at the range $3.68-4.72 \mathrm{ppm}$ as a result of closed $\mathrm{N}$ with drawing atom effect. The $\mathrm{CH}(2 \& 2$ ') show a pentate signals at the range $1.4-2.72 \mathrm{ppm}$, while $\mathrm{CH}$ (3 \& 3') show also pentane or multiplane signals at the range $1.2-1.56 \mathrm{ppm}$. NH proton gave a signal at 7.2- $8.3 \mathrm{ppm}$ as a singlet band that is confirm with the proposed structures, see table $3 \mathrm{a}$ and $\mathrm{b}$ ).

Table 3. ${ }^{1} \mathrm{H}$ NMR data for some new polymers

\begin{tabular}{|c|c|c|}
\hline $\begin{array}{l}\text { Compd. } \\
\text { no. }\end{array}$ & Compound structure as in NMR & Chemical shifts (ppm) \\
\hline II & & $\begin{array}{c}\text { 9.60(s) SH (tautomerism) ; } 8.19(\mathrm{~s}), 7.87(\mathrm{~s}) \mathrm{NH} ; \\
\text { 4.33(q), 3.62(q), 3.14(q) } \mathrm{CH}_{1}, \mathrm{CH}_{1}, ; 1.73(\mathrm{p}) \\
1.57(\mathrm{p}), 1.26(\mathrm{p}) \mathrm{CH}_{2}\end{array}$ \\
\hline III & 3 & $\begin{array}{l}7.92(\mathrm{~s}) \mathrm{NH} ; 4.32(\mathrm{q}), 4.20(\mathrm{q}) \mathrm{CH}_{1}, \mathrm{CH}_{1^{\prime}} \\
1.48(\mathrm{p}), \mathrm{CH}_{2}, \mathrm{CH}_{2^{\prime}} ; 1.24(\mathrm{p}) \mathrm{CH}_{3}, \mathrm{CH}_{3^{\prime}}\end{array}$ \\
\hline 1 & & 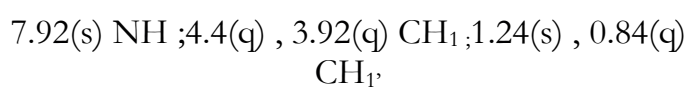 \\
\hline 2 & & $\begin{array}{c}7.87(\mathrm{~s}) \mathrm{NH} ; 3.82(\mathrm{q}), 3.10(\mathrm{q}) \mathrm{CH}_{1}, \mathrm{CH}_{1}, ; 1.96(\mathrm{p}) \\
, 1.70(\mathrm{p}), 1.25(\mathrm{p}) \mathrm{CH}_{2}\end{array}$ \\
\hline 3 & & $\begin{array}{c}\text { 7.68(s) } \mathrm{NH} ; 4.32(\mathrm{q}), 3.68(\mathrm{q}) \mathrm{CH}_{1}, \mathrm{CH}_{1^{\prime}} \\
2.67(\mathrm{p}), \mathrm{CH}_{2}, \mathrm{CH}_{2^{\prime}} \\
1.80,1.32(\mathrm{p}) \mathrm{CH}_{3}, \mathrm{CH}_{3^{\prime}}\end{array}$ \\
\hline 4 & & $\begin{array}{c}\text { 8.32(s) } \mathrm{NH} ; 3.92(\mathrm{~s}), 2.60(\mathrm{q}) \mathrm{CH}_{1}, \mathrm{CH}_{1}, 2.32(\mathrm{~d}) \\
\mathrm{CH}_{3} \mathrm{Si}, \mathrm{CH}_{2}, 2.20(\mathrm{t}) \\
\text { another config. }(\mathrm{NH}-\mathrm{Si})\end{array}$ \\
\hline 5 & & $\begin{array}{c}7.86(\mathrm{~s}) \mathrm{NH} ; 3.76(\mathrm{q}), 3.60(\mathrm{q}) \mathrm{CH}_{1}, \mathrm{CH}_{1} ; 1.97(\mathrm{p}) \\
1.59(\mathrm{p}), 1.16(\mathrm{p}) \mathrm{CH}_{2} ; 3.04(\mathrm{~s}) \mathrm{Si}_{-} \mathrm{CH}_{3}\end{array}$ \\
\hline 6 & & $\begin{array}{l}\text { 7.65(s), 7.24(s) } \mathrm{NH} ; 4.48(\mathrm{q}) \\
3.60(\mathrm{q}) \mathrm{CH}_{1}, \mathrm{CH}_{1^{\prime}} ; 1.6,1.4(\mathrm{p}), \mathrm{CH}_{2}, \mathrm{CH}_{2^{\prime}} \\
; 1.24(\mathrm{~m}) \mathrm{CH}_{3}, \mathrm{CH}_{3^{\prime}}, 4,4^{\prime} .\end{array}$ \\
\hline 7 & & $\begin{array}{c}7.36(\mathrm{~s}) \mathrm{NH} ; 4.36(\mathrm{q}), 4.20(\mathrm{q}) \mathrm{CH}_{1}, \mathrm{CH}_{1^{\prime}} ; \\
2.76(\mathrm{p}), 1.52(\mathrm{~m}) \mathrm{CH}_{2}, \mathrm{CH}_{2^{\prime}} ; 1.32,1.20(\mathrm{~m}) \mathrm{CH}_{3} \\
{ }_{,} \mathrm{CH}_{3^{\prime}}\end{array}$ \\
\hline 9 & & $\begin{array}{c}\text { 7.92(s) } \mathrm{NH} ; 4.72(\mathrm{q}), \mathrm{CH}_{1}, \mathrm{CH}_{1^{\prime}} ; \\
1.48(\mathrm{p}), \mathrm{CH}_{2}, \mathrm{CH}_{2^{\prime}} ; 1.24(\mathrm{p}) \mathrm{CH}_{3}, \mathrm{CH}_{3^{\prime}}\end{array}$ \\
\hline 10 & & $\begin{array}{c}8.1(\mathrm{~s}) \mathrm{NH} ; 4.08(\mathrm{q}), 3.68(\mathrm{q}) \mathrm{CH}_{1}, \mathrm{CH}_{1^{\prime}} ; \\
2.80(\mathrm{p}), 1.60 \text { (p) } \mathrm{CH}_{2}, \mathrm{CH}_{2^{\prime}} ; 1.561 .26(\mathrm{p}) \mathrm{CH}_{3} \\
\mathrm{CH}_{3^{\prime}}\end{array}$ \\
\hline 11 & & $\begin{array}{l}\text { 7.6(s) } \mathrm{NH} ; 4.4(\mathrm{~m}), 2.8(\mathrm{~m}) \mathrm{CH}_{1}, \mathrm{CH}_{1^{\prime}} ; 1.68,1.56 \\
\quad(\mathrm{~m}), \mathrm{CH}_{2}, \mathrm{CH}_{2^{\prime}} ; 1.4,1.26(\mathrm{~m}) \mathrm{CH}_{3}, \mathrm{CH}_{3^{\prime}}\end{array}$ \\
\hline
\end{tabular}




\section{THERMAL STUDY:}

TGA:

The main interest in this article included the study of the behaviour of prepared new polymers and their epoxy derivatives against high temperatures in order to use them as a coating polymers in agriculture studies. All prepared polymers even sodium salts may show activity as an anti-fungal that is because zinc polymers with (ethylene bisdithiocarbamate and 1,2-ethylene bis- dithiocarbamate are well known as a fungicides $\stackrel{(7)}{ }$.

Sodium salts of dithiocarbamate undergoes a thermal degradation. A same pattern of decomposition steps starting with the loosing of water molecules at about $\left(70-160{ }^{\circ} \mathrm{C}\right)$ followed by carbon disulphide and metals, that is support hardly the proposed structures. See scheme (2) below.

The TGA analysis figure for silicon polymer show the loosing of water molecules followed by dimethylsilane in two steps with a high residue up to about $68.31 \%$ at $800^{\circ} \mathrm{C}$.

The thermal degradation of tellurium and transition metal polymers began at about $100^{\circ} \mathrm{C}$ by losing water molecules and almost complete at $300{ }^{\circ} \mathrm{C}$ with a mass loss of halogen and metal halides with a chare contain equal to about $30 \%$ that is confirm with previous studies*, see Schemes below .

\section{Compound No. II}

$$
\begin{aligned}
& \mathrm{NaS}_{2} \mathrm{CNH}\left(\mathrm{CH}_{2}\right)_{3} \mathrm{NHCS}_{2} \mathrm{Na} .4 \mathrm{H}_{2} \mathrm{O} \stackrel{-4 \mathrm{H}_{2} \mathrm{O}}{100^{\circ} \mathrm{C}} \underset{\left.\begin{array}{l}
\text { Calculated : } 78.9 \% \\
\text { NaS }
\end{array} \mathrm{CNH}_{2} \mathrm{CH}_{2}\right)_{3} \mathrm{NHCS}_{2} \mathrm{Na}}{\text { Calnd }} \\
& \text { Found } \quad: 79.0 \% \\
& \mathrm{NaS}_{2} \mathrm{CNH}\left(\mathrm{CH}_{2}\right)_{3} \mathrm{NHCS}_{2} \mathrm{Na} \frac{-\mathrm{CH}_{3} \mathrm{CH}_{2} \mathrm{NHCS}_{2} \mathrm{Na}}{223{ }^{\circ} \mathrm{C}} \mathrm{CH}_{3} \mathrm{NHCS}_{2} \mathrm{Na} \\
& \text { Calculated : } 37.72 \% \\
& \mathrm{CH}_{3} \mathrm{NHCS}_{2} \mathrm{Na} \stackrel{-\mathrm{CH}_{3} \mathrm{CH}_{2} \mathrm{NH}}{454.5^{\circ} \mathrm{C}} \underset{\substack{\text { Calculated }: 22.8 \% \\
\mathrm{Na}_{2} \mathrm{~S}}}{\text { Found }} \\
& \text { Found : } 23.6 \%
\end{aligned}
$$

\section{Compound No. III}

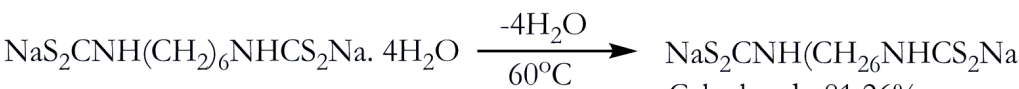

$$
\begin{aligned}
& \text { Calculated : } 81.26 \% \\
& \text { Found } \quad: 81.0 \% \\
& \mathrm{NaS}_{2} \mathrm{CNH}\left(\mathrm{CH}_{2}\right)_{6} \mathrm{NHCS}_{2} \mathrm{Na} \underset{136^{\circ} \mathrm{C}}{\stackrel{-\mathrm{NaS}}{\longrightarrow}} \begin{array}{l}
\mathrm{NaS}_{2} \mathrm{CNH}\left(\mathrm{CH}_{2}\right)_{6} \mathrm{NHCS} \\
\text { Calculated }: 67.20 \%
\end{array} \\
& \text { Found : } 66.80 \%
\end{aligned}
$$

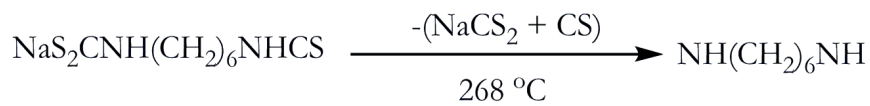

$$
\begin{aligned}
& \text { Calculated : } 30.22 \% \\
& \text { Found } \quad: 31.0 \% \\
& \mathrm{NH}\left(\mathrm{CH}_{2}\right)_{6} \mathrm{NH} \underset{391^{\circ} \mathrm{C}}{-\mathrm{CH}_{3} \mathrm{NH}} \\
& \text { Calculated : } 18.52 \% \\
& \text { Found } \quad: 18.82 \%
\end{aligned}
$$


Compound No. 2

$$
\begin{aligned}
& {\left[( \mathrm { CH } _ { 3 } ) _ { 2 } \mathrm { Si } _ { 2 } \mathrm { S } _ { 2 } \mathrm { CNH } ( \mathrm { CH } _ { 2 } ) _ { 6 } \mathrm { NHCS } _ { 2 } [ \mathrm { Si } ( \mathrm { CH } _ { 3 } ) _ { 2 } ] _ { 2 } . 2 \mathrm { H } _ { 2 } \mathrm { O } \underset { 2 } { \stackrel { - 2 \mathrm { H } _ { 2 } \mathrm { O } } { 2 1 0 } \mathrm { C } } \left[\left(\mathrm{CH}_{3}\right)_{2} \mathrm{Si}_{2} \mathrm{~S}_{2} \mathrm{CNH}\left(\mathrm{CH}_{2}\right)_{6} \mathrm{NHCS}_{2}\left[\mathrm{Si}\left(\mathrm{CH}_{3}\right)_{2}\right] 2 .\right.\right.} \\
& \text { Calculated : } 91.37 \% \\
& \text { Found } \quad: 92.27 \%
\end{aligned}
$$

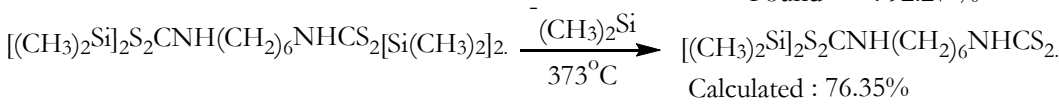

$$
\begin{aligned}
& \text { Found : } 74.98 \%
\end{aligned}
$$

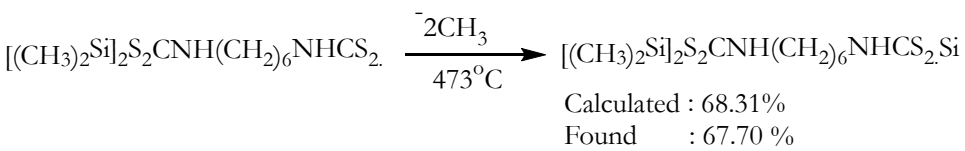

\section{Compound No. 4}

$$
\begin{aligned}
& \mathrm{Cl}_{2} \mathrm{NiS}_{2} \mathrm{CNH}\left(\mathrm{CH}_{2}\right)_{6} \mathrm{NHCS}_{2} \mathrm{NiCl}_{2} 4 \mathrm{H}_{2} \mathrm{O} \underset{-\mathrm{Cl} 131^{\circ} \mathrm{C}}{-} \mathrm{Cl}_{2} \mathrm{NiS}_{2} \mathrm{CNH}\left(\mathrm{CH}_{2}\right)_{6} \mathrm{NHCS}_{2} \mathrm{NiCl} \\
& \text { Calculated : } 82.01 \% \\
& \text { Found } \quad: 82.0 \%
\end{aligned}
$$

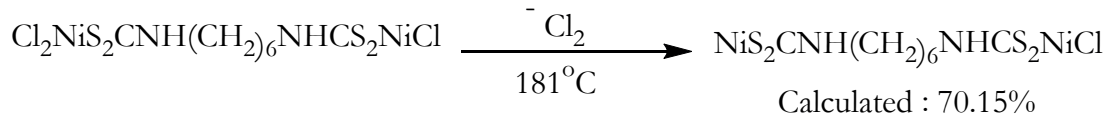

$$
\begin{aligned}
& \text { Found : } 70.2 \%
\end{aligned}
$$

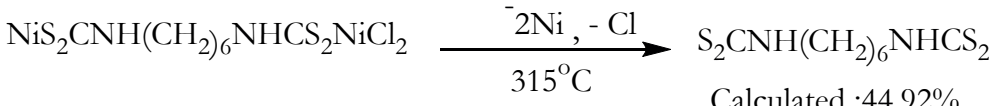

$$
\begin{aligned}
& \text { Found } \quad: 44.5 \% \\
& \mathrm{~S}_{2} \mathrm{CNH}\left(\mathrm{CH}_{2}\right)_{6} \mathrm{NHCS}_{2} \underset{{ }^{-} \mathrm{NHCS}_{2}}{\stackrel{{ }^{\circ}}{\longrightarrow}} \stackrel{\mathrm{S}_{2} \mathrm{CNH}\left(\mathrm{CH}_{2}\right)_{6} \mathrm{C}}{\text { Calculated }: 29.67 \%} \\
& \text { Found } \quad: 29.7 \%
\end{aligned}
$$

\section{Compound No. 5}

$$
\begin{aligned}
& \mathrm{Cl}_{2} \mathrm{CoS}_{2} \mathrm{CNH}\left(\mathrm{CH}_{2}\right)_{6} \mathrm{NHCS}_{2} \mathrm{CoCl}_{2} 4 \mathrm{H}_{2} \mathrm{O} \frac{-2 \mathrm{H}_{2} \mathrm{O}}{128^{\circ} \mathrm{C}} \longrightarrow \mathrm{Cl}_{2} \mathrm{CoS}_{2} \mathrm{CNH}\left(\mathrm{CH}_{2}\right)_{6} \mathrm{NHCS}_{2} \mathrm{CoCl}_{2} 2 \mathrm{H}_{2} \mathrm{O} \\
& \text { Calculated : } 93.76 \%
\end{aligned}
$$

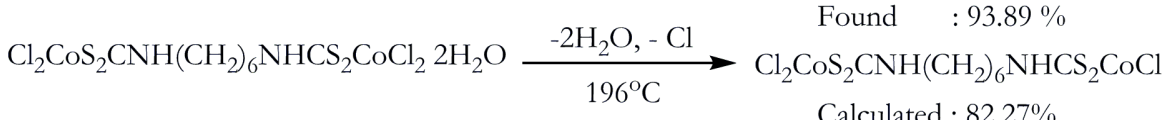

$$
\begin{aligned}
& \text { Found } \quad: 82.278 \% \\
& \mathrm{Cl}_{2} \mathrm{CoS}_{2} \mathrm{CNH}\left(\mathrm{CH}_{2}\right)_{6} \mathrm{NHCS}_{2} \mathrm{CoCl}_{2} 2 \mathrm{H}_{2} \mathrm{O} \underset{308^{\circ} \mathrm{C}}{\stackrel{-\mathrm{Co} \mathrm{ClCS}_{2}}{\longrightarrow}} \mathrm{NH}\left(\mathrm{CH}_{2}\right)_{6} \mathrm{NHCS}_{2} \mathrm{CoCl}_{2} \\
& \text { Found : } 54.96 \%
\end{aligned}
$$

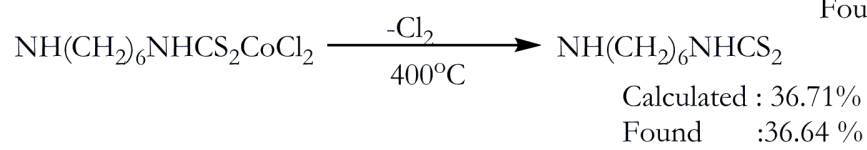




\section{Compound No. 7}

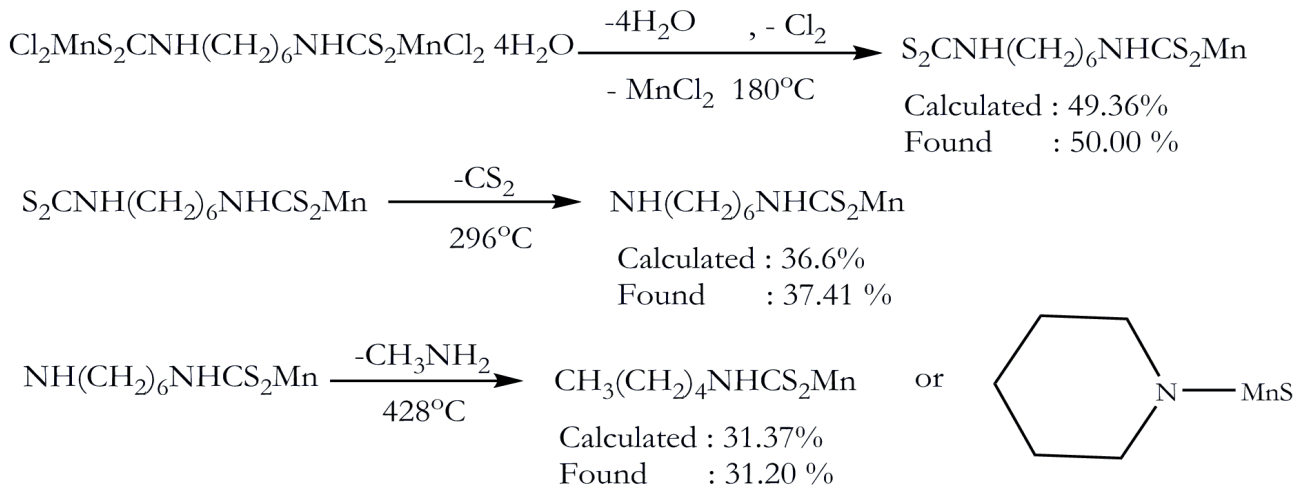

\section{Compound No. 8}

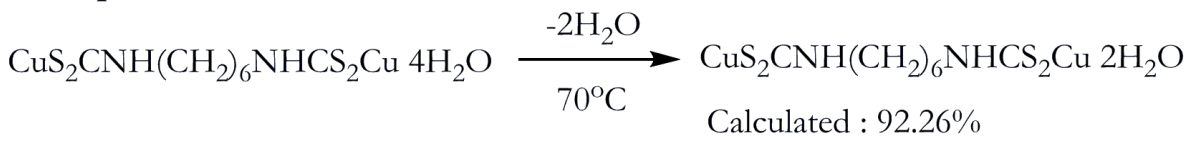

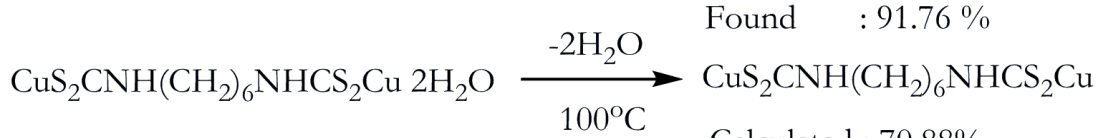

Calculated : $70.88 \%$

Found $\quad: 70.50 \%$

$\mathrm{CuS}_{2} \mathrm{CNH}\left(\mathrm{CH}_{2}\right)_{6} \mathrm{NHCS}_{2} \mathrm{Cu} \underset{-\mathrm{CNH}\left(\mathrm{CH}_{2}\right)_{6} \mathrm{NHCS}_{2}}{264^{\circ} \mathrm{C}} \mathrm{Cu}_{2} \mathrm{~S}_{2}$

Calculated : $41.06 \%$

Found : $41.18 \%$

\section{Compound No. 9}

$\mathrm{Br}_{2} \mathrm{TeS}_{2} \mathrm{CNH}\left(\mathrm{CH}_{2}\right)_{6} \mathrm{NHCS}_{2} \mathrm{TeBr}_{2} 4 \mathrm{H}_{2} \mathrm{O} \underset{212^{\circ} \mathrm{C}}{\stackrel{-4 \mathrm{H}_{2} \mathrm{O},-\mathrm{TeBr}_{4}}{\longrightarrow}} \mathrm{TeS}_{2} \mathrm{CNH}\left(\mathrm{CH}_{2}\right)_{6} \mathrm{NHCS}_{2}$ Calculated : $39.42 \%$

Found : $38.89 \%$

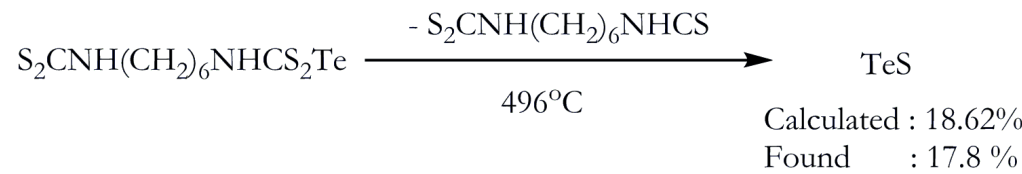

\section{Compound No. 11}

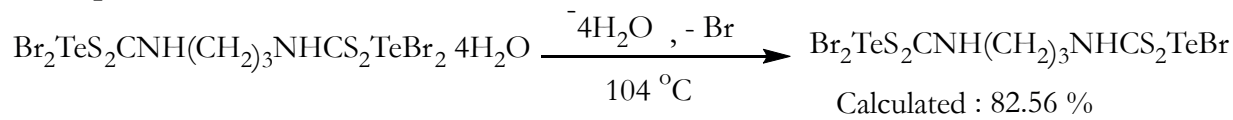

$$
\text { Found } \quad: 82.57 \%
$$

$\mathrm{Br}_{2} \mathrm{TeS}_{2} \mathrm{CNH}\left(\mathrm{CH}_{2)}{ }_{3} \mathrm{NHCS}_{2} \mathrm{TeBr} \underset{196{ }^{\circ} \mathrm{C}}{\stackrel{-\mathrm{Br}_{2}}{\longrightarrow}} \underset{\text { Calculated }: 64.22 \%}{\mathrm{TeS}_{2} \mathrm{CNH}\left(\mathrm{CH}_{2}\right)_{3} \mathrm{NHCS}_{2} \mathrm{TeBr}}\right.$

Found : $64.93 \%$

$\mathrm{TeS}_{2} \mathrm{CNH}\left(\mathrm{CH}_{2}\right)_{3} \mathrm{NHCS}_{2} \mathrm{TeBr} \underset{367{ }^{\circ} \mathrm{C}}{\stackrel{-\mathrm{Te}}{\longrightarrow}} \mathrm{S}_{2} \mathrm{CNH}\left(\mathrm{CH}_{2}\right)_{3} \mathrm{NHCS}_{2} \mathrm{TeBr}$

Calculated : $49.69 \%$
Found $\quad: 50.50 \%$

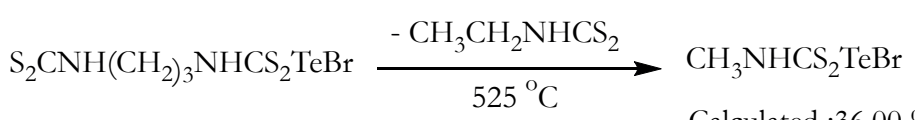

Calculated : $36.00 \%$

Found : $36.63 \%$ 


\section{DSC:}

The differential scanning calorimetry (DSC) for new polymers have been studied, commercial epoxy resin was used. The study gave a confirm results with zineb (zinc ethylene bis-dithiocarbamate) that is used as a fungi side and triethyltetraamine as a commercial resin.

Silicone and Tellurium polymers gave glass transitions at $\left(\mathrm{Si}-\mathrm{H}, 1^{\text {st }} 63.93,2^{\text {nd }} 84.73 ;\left(\mathrm{Te}-\mathrm{H}, 1^{\text {st }} 30.58\right.\right.$ $, 2^{\text {nd }} 64.87,3^{\text {rd }}, 450 ;\left(\mathrm{Zn}-\mathrm{H}, 1^{\text {st }} 60.91,2^{\text {nd }} 75.33\right.$; $\left(\right.$ Si-P, $1^{\text {st }} 58.47,2^{\text {nd }} 78.83 ;\left(\right.$ Si-E, $1^{\text {st }} 60.38,2^{\text {nd }} 77.29$ ). Silicone polymers data are closed to that of zinc polymer while tellurium polymer gave low glass transition compared with other that is may due to atomic radius and flexibility in molecular movement see figures (2a-2f). The other metal polymers gave one glass transition (Cd , 160; Co, 210 ; Cu, 285) that is may due to vibration of polymer chains.

\section{Epoxy resins:}

Two characteristic absorption of the oxirane ring are observed in the range between 4000 and $400 \mathrm{~cm}^{-1}$. The first one, at $915 \mathrm{~cm}^{-1}$, is attributed to the $\mathrm{C}-\mathrm{O}$ deformation of the oxidant group, the second band is located at $3050 \mathrm{~cm}^{-1}$ approximately and is attributed to the $\mathrm{C}-\mathrm{H}$ tension of the methylene group of the epoxy ring. This band is not very useful since its intensity is low and it is very close to the strong $\mathrm{O}-\mathrm{H}$ absorptions $\frac{(8)}{\text {. }}$.

The study showed that the new silicone polymers act as a stable polymers compared with others. Table (4) based on the rate of thermal dissociation of selected polymers, in this table, all new polymers are of a high stability with large values of char contain compared with commercial epoxy. Among silicone polymers, the polymer with carbon chain equal to 3 is more stable than that with 2 carbon atoms while the silicone polymer with 6 carbon atoms is the less stable one, may thermal treatment caused decomposition combined the forming cyclic compound with a tetramer dimethyl silane. the sequence of stability as shown below:

TePdtc Zn Edtc SiEdtc SiPdtc TeEdtc SiHdtc Where $($ Edtc=ethelenebis-dithiocarbamate;P$\mathrm{dtc}=$ propelenebis-dithiocarbamate $;$ Hdtc $=$ hexamethelene- bis-dithiocarbamate), see Figure ( $3 a$ and b).

Table 4. TGA data for commercial and new epoxides of selected new polymer

\begin{tabular}{ccccc}
\hline $\begin{array}{l}\text { Hardener name } \\
\text { (Compd. no. }\end{array}$ & D.T & Char $\%$ at $650^{\circ} \mathrm{C}$ & Tem. of $50 \%$ wt. loss & Rate of decomp. \%/min \\
\hline TETA & $500{ }^{\circ} \mathrm{C}$ & $40 \%$ & $525^{\circ} \mathrm{C}$ & 4.80 \\
Si- HDTC (6) & $499{ }^{\circ} \mathrm{C}$ & $64 \%$ & $>700{ }^{\circ} \mathrm{C}$ & 2.88 \\
Te- EDTC (1) & $497{ }^{\circ} \mathrm{C}$ & $65 \%$ & $>700{ }^{\circ} \mathrm{C}$ & 2.80 \\
Si- EDTC (4) & $490{ }^{\circ} \mathrm{C}$ & $66 \%$ & $>700{ }^{\circ} \mathrm{C}$ & 2.72 \\
Zn- EDTC (7) $_{\text {Ei- PDTC (5) }}$ & $488^{\circ} \mathrm{C}$ & $67 \%$ & $>700{ }^{\circ} \mathrm{C}$ & 2.64 \\
\hline
\end{tabular}




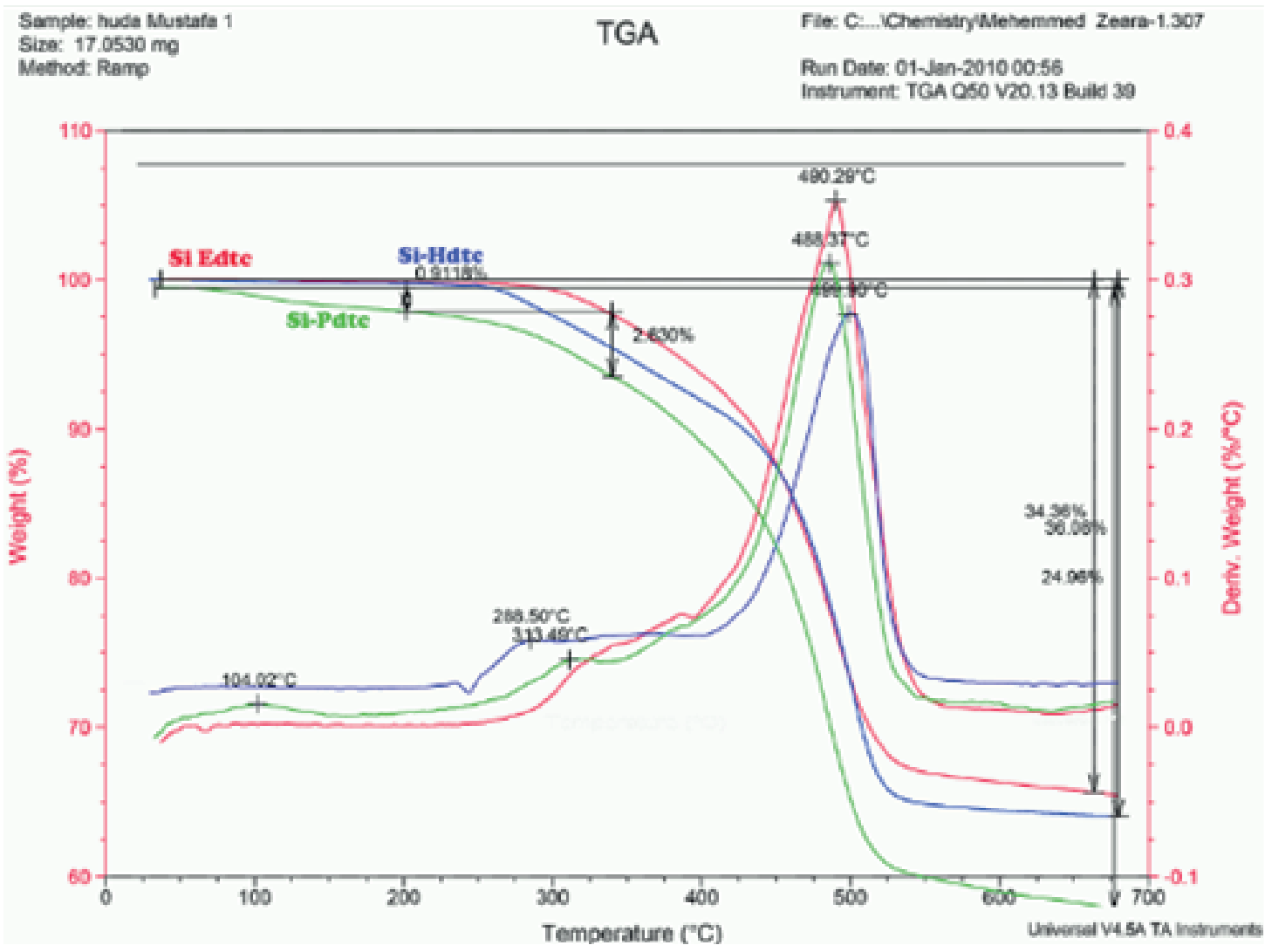

Figure (3a). TGA curve for Si hardener with Epoxy

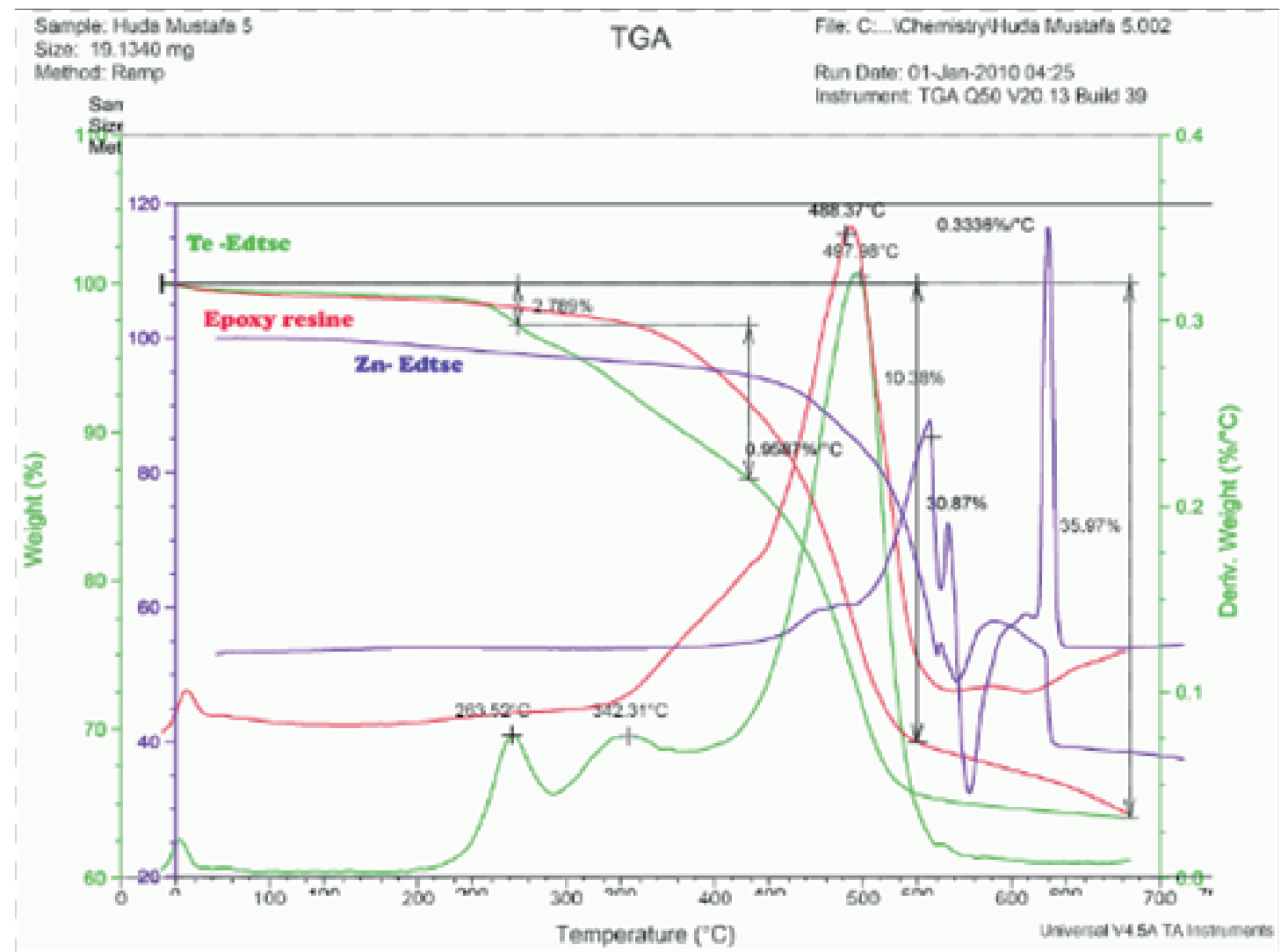

Figure (3b). TGA curve for Zn-Edtc polymer, Te-Edtc and commercial Epoxy 


\section{CONCLUSIONS}

A new series of polymers based on bisdithiocarbamate ligands were prepared and characterized with several techniques. The study showed that the polymers of square planner or tetrahedral configuration. The new polymers are insoluble in common organic solvents. They are soluble in DMSO. All these new polymers showed a good thermal Stability and used as a hardener for epoxy resins.

\section{REFERENCES}

1. .Ning T, Xinmin G, Hailiane $Z$ and Minyu $T$, Pollyhedron, 1990; $9: 859-862$. https://doi.org/10.1016/S0277-5387(00)81352-2

2. Baroncini E. A, Yadav S. K., Palmese G. R, Stanzione J. F.; REVIEW J. APPL. POLYM. SCI. 2016; 44103: 1 - 19
3. Ahmed MAK., McWinnie W R and Granger P., Polyhedron 1986;5:859-862. https://doi.org/10.1016/S0277-5387(00)84448-4

4. Huang Z, Lakshmikantham M V , Lyon M, Cava M P, J. Org. Chem., 2000;65: 3413 https://doi.org/10.1021/jo0001030

5. Edward R.T, Zukerman J, Coor. Chem. Rev. 2010; 46: 254.

6. Al-Rubaie A Z and Al-Luaibi M Y, Int.J. Sci. 2005; 14: 71-78.

7. Müller F, Ackermann P, Margot P "Fungicides, Agricultural, Individual Fungicides". Weinheim: Wiley-VCH. doi:10.1002/14356007, 2012; 2. . https://doi.org/10.1002/14356007

8. Shreve O D, Anal. Chem., 1952; 24 (11): 16921699. https://doi.org/10.1021/ac60071a003

9. Rosu D, Mustata F. . Cascaval CN, Thermochimica Acta ,2001;370(1-2):105-110. https://doi.org/10.1016/S0040-6031(00)00787-5 